\title{
Binary blends of diblock copolymers as an effective route to multiple length scales in perfect directed self-assembly of diblock copolymer thin films
}

\author{
Erik W. Edwards, Mark P. Stoykovich, and Paul F. Nealey ${ }^{a)}$ \\ Department of Chemical and Biological Engineering, University of Wisconsin-Madison, Madison, Wisconsin \\ 53706 and Center for Nanotechnology, University of Wisconsin-Madison, Madison, Wisconsin 53706 \\ Harun H. Solak \\ Laboratory for Micro and Nanotechnology, Paul Scherrer Institut, Villigen-PSI, Switzerland CH-5232
}

(Received 2 June 2005; accepted 14 November 2005; published 25 January 2006)

\begin{abstract}
The directed assembly of binary blends of diblock copolymers on chemically nanopatterned substrates was investigated as a means to pattern features of controlled dimensions at the nanoscale. The lamella-forming blends assembled without defects and in registration with underlying chemical surface patterns that had periods $L_{S}$ that were commensurate with the bulk lamellar period of the blends $L_{B}$. $L_{B}$ was tuned between the bulk lamellar periods of the block copolymers $L_{O 1}$ and $L_{O 2}$. (C) 2006 American Vacuum Society. [DOI: 10.1116/1.2151226]
\end{abstract}

\section{INTRODUCTION}

Diblock copolymers are molecules that consist of two chemically distinct chains covalently bonded at one end. ${ }^{1}$ When heated above the glass transition temperatures of the constituent blocks and below an order-disorder transition temperature, diblock copolymers spontaneously microphase separate to form ordered structures including spheres, cylinders, and lamellae with characteristic lattice periods on the order of $10-50 \mathrm{~nm}$. The specific morphology and lattice period formed upon microphase separation depends on the relative volume fraction of the two blocks of the block copolymer and the overall molecular weight of the block copolymer chain. In thin films these materials can be used as templates for nanofabrication when one of the blocks of the diblock copolymer is selectively removed, a process known as block copolymer lithography. ${ }^{2}$ Block copolymer lithography has already been used to fabricate a variety of functional devices at the nanoscale, including quantum dots, ${ }^{2,3}$ increased capacitance gate devices, ${ }^{4,5}$ nanowires, ${ }^{6,7}$ magnetic storage media, ${ }^{8}$ and photonic crystals. ${ }^{9}$ Many next-generation applications will require perfect long-range ordering in the block copolymer domains. Moreover, some future applications will require registration of the individual block copolymer domains with respect to the underlying substrate. These requirements have resulted in the development of numerous methods to obtain long-range ordering and substrate registration of block copolymer domains. ${ }^{10-18}$

Previously, we have demonstrated that directed assembly of block copolymer domains on chemically nanopatterned substrates is a technique capable of forming defect-free periodic ${ }^{11,19}$ and nonregular device-oriented arrays ${ }^{20}$ of block copolymer domains in registration with the underlying chemical surface pattern. One characteristic of this technique is that perfect ordering of the block copolymer domains re-

a) Author to whom correspondence should be addressed; electronic mail: nealey@engr.wisc.edu quires the substrate pattern period $L_{S}$ to be commensurate with the bulk lamellar period of the block copolymer $L_{O}$. Furthermore, the range of substrate pattern periods (and thus the range of commensurability) that can direct the assembly of block copolymer domains to perfection is highly dependent on the strength of the interactions between the chemically nanopatterned regions of the substrate and the blocks of the block copolymer. ${ }^{19}$ There are two main strategies available for adjusting $L_{O}$ so as to create features of a given dimension, $L_{S}$ : (1) synthesizing a specific molecular-weight block copolymer for every desired feature size or (2) blending block copolymers with homopolymers, or other diblock copolymers, to shift the bulk lattice period of the block copolymer domains.

In bulk systems the repeat period of binary blends of diblock copolymers has been demonstrated to scale with the number average molecular weight of the blend to the $2 / 3$ power, as shown in Eq. (1): $:^{21,22}$

$$
L_{B} \sim\left(\Sigma x_{i} M_{N_{i}}\right)^{2 / 3},
$$

where $L_{B}$ is the bulk repeat period of the blend and $x_{i}$ and $M_{N_{i}}$ are the mole fraction and number average molecular weight of the $i$ th block copolymer chain, respectively. In this article we investigate the efficacy of using binary blends of symmetric, lamellar-forming diblock copolymers to change the substrate pattern period over which perfect directed selfassembly is observed. We find that the dimensions of defectfree lamellar domains on chemically nanopatterned substrates can be shifted over a range of pattern periods $L_{S}$ between two block copolymer bulk lamellar periods $L_{O 1}$ and $L_{O 2}$.

\section{EXPERIMENT}

Chemically nanopatterned substrates were prepared in the following manner: A 40-nm-thick layer of hydroxyterminated polymer was spin coated on silicon wafers that were cleaned with piranha solution $\left(7: 3 \mathrm{v} / \mathrm{v}_{2} \mathrm{O}_{2}: \mathrm{H}_{2} \mathrm{SO}_{4}\right.$, 
$100{ }^{\circ} \mathrm{C}, 30 \mathrm{~min}$ ). (Caution: piranha reacts violently with organic compounds and should not be stored in closed containers.) The silicon wafers coated with hydroxy-terminated polymer were then annealed at $160{ }^{\circ} \mathrm{C}$ for $48 \mathrm{~h}$ under vacuum to graft a $4 \mathrm{~nm}$ layer of polymer, henceforth referred to as a brush, to the silicon substrate. ${ }^{19,23}$ The excess, ungrafted hydroxy-terminated polymer was then removed by repeated sonication in warm toluene. The wafers with brushes were then spin coated with photoresist grade polymethyl methacrylate $\left(M_{N}=950 \mathrm{~kg} / \mathrm{mol}, 1.2 \%\right.$ in chlorobenzene, Microchem Corp., Newton, MA) to give films that were $50 \mathrm{~nm}$ thick. Following a post-apply bake at $130^{\circ} \mathrm{C}$ for $120 \mathrm{~s}$ the photoresist was patterned using extreme ultraviolet interferometric lithography (EUV-IL) as described previously in the literature. ${ }^{24}$ The EUV-IL technique involves patterning the photoresist by exposing a transmission membrane grating to EUV light. This generates an interference pattern at the substrate that has a period equal to half the grating period. In this study the transmission membrane grating yielded patterns in the photoresist consisting of lines and spaces of equal widths with periods $L_{S}$ between 40 and $50 \mathrm{~nm}$. EUV exposures were performed in vacuum at $10^{-5}$ mbar at the x-ray interference lithography beamline of the Swiss Light Source. The beamline uses undulator light with a central wavelength of $13.4 \mathrm{~nm}, 92 \mathrm{eV}$, and $4 \%$ spectral bandwidth. After exposure the samples were developed for $30 \mathrm{~s}$ in a 3:1 isopropyl alcohol:methyl isobutyl ketone developing solution, rinsed extensively with isopropyl alcohol, and blown dry in a stream of nitrogen. The topographic pattern in the photoresist was then transferred to a chemical pattern in the polymer brush by employing a $10 \mathrm{~s}$ oxygen plasma etch, which incorporated highly polar, oxygencontaining moieties in the portions of the polymer brush that were not protected by photoresist. ${ }^{25}$ The plasma was created with an oxygen flow rate of 8 sccs (standard cubic centimeters per second) and an applied voltage of $100 \mathrm{~V}$. The remaining photoresist structures were then stripped using sonication in warm chlorobenzene, yielding a chemically nanopatterned substrate consisting of stripes of polymer brush that alternated with stripes of the oxygenated polymer brush.

Two different polymer brushes, which have been previously characterized in the literature, ${ }^{19}$ were used in this study. The first brush was formed from hydroxy-terminated random copolymers containing $58 \%$ styrene and $42 \%$ methyl methacrylate by volume and is termed a "neutral brush" because the interfacial energy between the styrene block of the block copolymer and this brush is equal to the interfacial energy between the methyl methacrylate block of the block copolymer and this brush. ${ }^{23}$ Thus, neither block of the block copolymer preferentially wets the neutral brush. The second brush was formed from hydroxy-terminated polystyrene and is termed a "polystyrene brush." The interfacial energy between the styrene block of the block copolymer and the polystyrene brush is much lower than the interfacial energy between the methyl methacrylate block of the block copolymer and the polystyrene brush. Thus, styrene preferentially wets
TABLE I. Composition and bulk repeat periods of block copolymer thin films used in this study.

\begin{tabular}{lccc}
\hline \hline & $\begin{array}{c}\text { Volume fraction of } M_{N}= \\
(50-b-54) \mathrm{kg} / \mathrm{mol} \text { block } \\
\text { copolymer }\end{array}$ & $\begin{array}{c}\text { Volume fraction of } M_{N}= \\
(25-b-26) \mathrm{kg} / \mathrm{mol} \text { block } \\
\text { copolymer }\end{array}$ & $\begin{array}{c}\text { Bulk repeat } \\
\text { period, } L \\
(\mathrm{~nm})\end{array}$ \\
\hline LO48 & 1.00 & 0.00 & 48.4 \\
LB44 & 0.75 & 0.25 & 44.6 \\
LB41 & 0.50 & 0.50 & 41.4 \\
\hline \hline
\end{tabular}

the polystyrene brush. The methyl methacrylate block of the block copolymer always preferentially wets the oxygenated polymer brushes. Due to the strong preferential wetting of the polystyrene brush by the polystyrene block of the block copolymer, the patterned polystyrene brushes are termed to have a higher interfacial energy contrast than the patterned neutral brushes.

Binary blends of two block copolymers of poly(styrene$b$-methyl methacrylate) (PS- $b$-PMMA) were created as follows. The polymers, purchased from Polymer Source Inc., Dorval Quebec, had molecular weights of (25-b26) $\mathrm{kg} / \mathrm{mol}$ and $(50-b-54) \mathrm{kg} / \mathrm{mol}$ with polydispersity indexes of 1.09 and 1.04, respectively, and bulk lamellar repeat periods of $L_{O 32}=32 \mathrm{~nm}$ and $L_{O 48}=48 \mathrm{~nm}$, respectively. Blends of these block copolymers, at various volume fractions, were prepared in solution and spin coated on the chemically nanopatterned substrates to give films that were approximately $42 \mathrm{~nm}$ thick. These films were then annealed for $72 \mathrm{~h}$ at $190{ }^{\circ} \mathrm{C}$ under vacuum (1-2 torr). The resulting domain morphologies were imaged in plan view using a LEO-1550 VP field-emission scanning electron microscope (SEM) at an accelerating voltage of $1 \mathrm{keV}$.

Information about the average domain spacing of the annealed block copolymer films was gathered from a twodimensional fast Fourier transform (2D-FFT) analysis of scanning electron micrographs. 2D-FFTs that were 512 $\times 512$ pixels were interpolated to a $1023 \times 1023$ pixel grid. The intensities of the FFT power spectra were then averaged azimuthally using 512 rings around the $1023 \times 1023$ grid to yield the average intensity of the FFT in $q$ space ( $q$ $=2 \pi / L)$.

\section{RESULTS}

The bulk lamellar periods of the blends used in this study were determined from a 2D-FFT analysis of scanning electron micrographs of the blends after annealing on unpatterned neutral brushes and are presented in Table $\mathrm{I}^{26}$ The first blend consisted of $25 \%$ of the $L_{O 32}=32 \mathrm{~nm}$ block copolymer and $75 \%$ of the $L_{O 48}=48 \mathrm{~nm}$ block copolymer and had a bulk lamellar period $L_{B 44}=44.6 \mathrm{~nm}$. The second blend consisted of $50 \%$ of the $L_{O 32}=32 \mathrm{~nm}$ block copolymer and $50 \%$ of the $L_{O 48}=48 \mathrm{~nm}$ block copolymer and had a bulk lamellar period $L_{B 41}=41.4 \mathrm{~nm}$. These blends are referred to as LB44 and LB41, respectively. The pure $L_{O 48}=48 \mathrm{~nm}$ block copolymer was also investigated in this study and is referred to as $\mathrm{LO} 48$. 


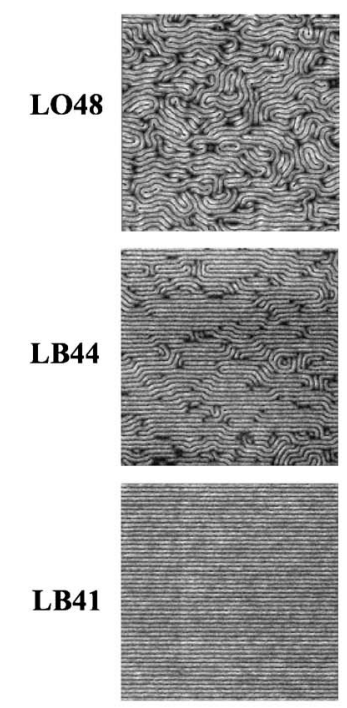

$\mathrm{L}_{\mathrm{S}}=40 \mathrm{~nm}$
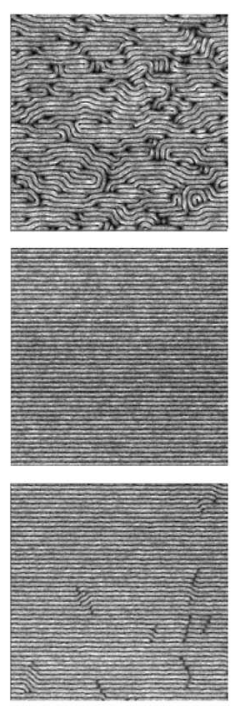

$\mathrm{L}_{\mathrm{S}}=42.5 \mathrm{~nm}$
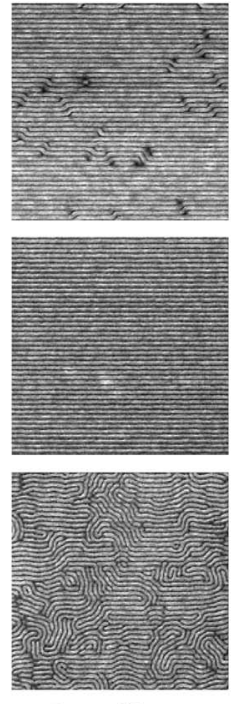

$\mathrm{L}_{\mathrm{S}}=45 \mathrm{~nm}$
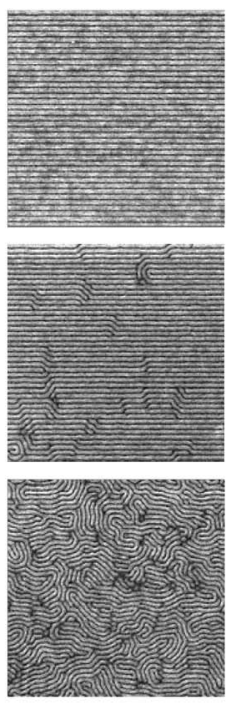

$\mathrm{L}_{\mathrm{S}}=47.5 \mathrm{~nm}$
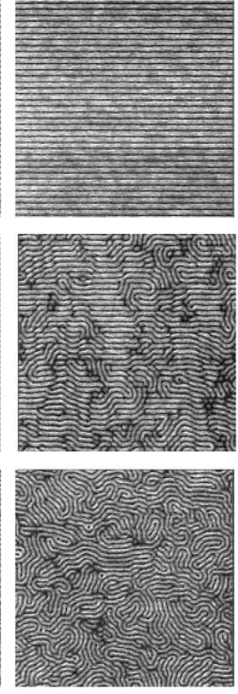

$\mathbf{L}_{\mathrm{S}}=\mathbf{5 0} \mathbf{~ \mathbf { m }}$
FIG. 1. Plan-view scanning electron micrographs of binary blends of diblock copolymers on chemically nanopatterned neutral polymer brushes. The period of the underlying chemical nanopattern increases from 40 to $50 \mathrm{~nm}$ from left to right in increments of $2.5 \mathrm{~nm}$. From top to bottom the rows contain micrographs of LO48, LB44, and LB41. The micrographs all image $2 \times 2 \mu \mathrm{m}^{2}$ areas.
Binary blends of PS- $b$-PMMA block copolymers enabled perfect directed assembly of the lamellar domains at substrate pattern periods between $L_{O 32}$ and $L_{O 48}$, as shown in the series of SEM images presented in Fig. 1. The SEM images capture the behavior of PS- $b$-PMMA domains after annealing on chemically nanopatterned neutral polymer brushes and are presented with the substrate pattern period increasing from left to right in $2.5 \mathrm{~nm}$ increments with a minimum pattern period of $40 \mathrm{~nm}$ and a maximum pattern period of $50 \mathrm{~nm}$. In the rows from top to bottom the SEM images capture the behavior of PS- $b$-PMMA domains of the LO48, LB44, and LB41 films. The pattern periods where perfect registration of the block copolymer domains occurred were commensurate with the bulk lamellar period of the block copolymer blends. For the neat block copolymer perfect registration occurred over the $L_{S}=47.5 \mathrm{~nm}$ and $L_{S}=50 \mathrm{~nm}$ pattern periods, in good agreement with our previously reported results. ${ }^{19}$ By adding $25 \%$ of the $L_{O 32}=32 \mathrm{~nm}$ block copoly- mer in LB44 the range of pattern periods over which defectfree domain registration occurred shifted to $L_{S}=42.5 \mathrm{~nm}$ and $L_{S}=45 \mathrm{~nm}$. As the fraction of $L_{O 32}=32 \mathrm{~nm}$ block copolymer was further increased to $50 \%$ in LB41, only the $L_{S}=40 \mathrm{~nm}$ chemical nanopatterns exhibited perfect domain registration.

Blends that were annealed on patterned polystyrene brushes, which had a higher interfacial energy contrast than the patterned neutral brushes, exhibited only marginal improvement in the registration of the block copolymer domains at noncommensurate length scales. Figure 2 presents a series of SEM images that capture the domain behavior in the blends after annealing on nanopatterned polystyrene brushes. For both blends the registration of the domains is slightly improved with respect to the registration observed in self-assembled blends on the chemically nanopatterned neutral brushes, with fewer dislocation and disclination defects appearing at substrate pattern periods that are incommensurate with the blend repeat period. However, the blends do not
LO48
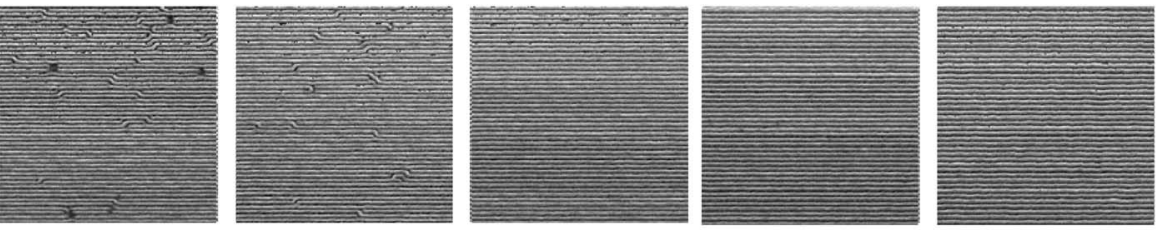

LB44
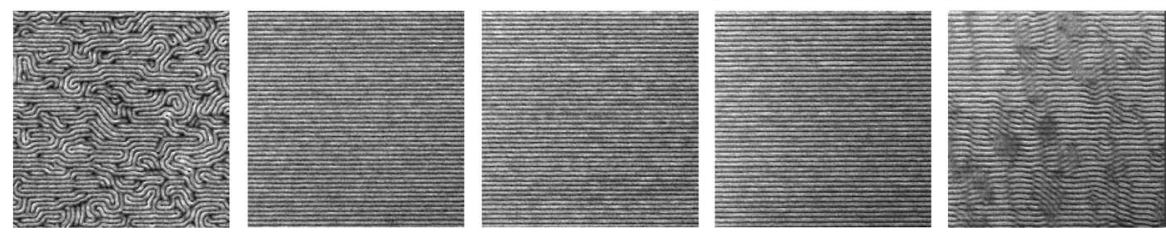

LB41
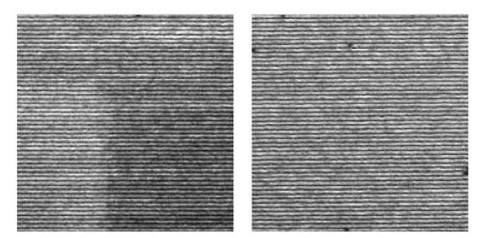

$\mathrm{L}_{\mathrm{S}}=40 \mathrm{~nm}$

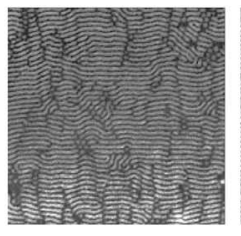

$\mathbf{L}_{\mathrm{S}}=45 \mathrm{~nm}$

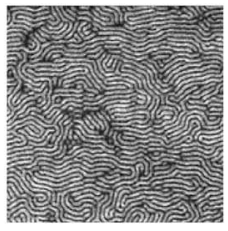

$\mathrm{L}_{\mathrm{S}}=47.5 \mathrm{~nm}$

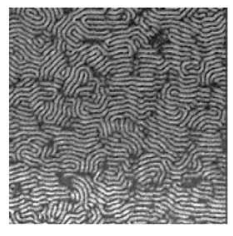

$\mathrm{L}_{\mathrm{S}}=\mathbf{5 0} \mathbf{n m}$
FIG. 2. Plan-view scanning electron micrographs of binary blends of diblock copolymers on chemically nanopatterned polystyrene brushes. The period of the underlying chemical nanopattern increases from 40 to $50 \mathrm{~nm}$ from left to right in increments of $2.5 \mathrm{~nm}$. From top to bottom the rows contain micrographs of LO48, LB44, and LB41. The micrographs all image $2 \times 2 \mu \mathrm{m}^{2}$ areas. 
exhibit an improvement in the registration of lamellar domains at highly incommensurate length scales.

Azimuthally averaged power spectra of 2D-FFTs of the SEM images presented in Figs. 1 and 2 confirm that the improvement of domain registration due to interfacial effects is suppressed in the binary blends of block copolymers. Figure 3 presents these spectra for each blend used in this study on both patterned polystyrene brushes and patterned neutral brushes. Whereas the azimuthally averaged power spectra of the 2D-FFT of the neat block copolymer $\left(L_{O 48}=48 \mathrm{~nm}\right)$ annealed on a patterned neutral brush with $L_{S}=40 \mathrm{~nm}$ was effectively identical to the power spectra of the disordered diblock copolymer used to calculate $L_{O}$, on patterned polystyrene brushes with $L_{S}=40 \mathrm{~nm}$ the azimuthally averaged power spectra of the 2D-FFT of the neat block copolymer $\left(L_{O 48}=48 \mathrm{~nm}\right)$ displayed the appearance of a well-defined narrow peak corresponding to the registration of the block copolymer domains with the underlying chemical surface pattern. Additionally, the peaks in the azimuthally averaged 2D-FFT power spectra had greater intensities and were narrower for the patterned polystyrene brushes on the $L_{S}$ $=42.5 \mathrm{~nm}, L_{S}=45 \mathrm{~nm}$, and $L_{S}=50 \mathrm{~nm}$ chemical surface patterns, as compared to the patterned neutral brushes. The binary blends of block copolymers demonstrated nearly identical azimuthally averaged 2D-FFTs for their domain behavior on patterned polystyrene brushes and on patterned neutral brushes, which indicated that any improvement of the domain registration due to interfacial energy effects was minimal in these blends.

\section{DISCUSSION}

These results are very promising with respect to making directed self-assembly of diblock copolymer domains further amenable to microelectronics manufacturing. The ability to address controllable length scales between the bulk lamellar period of two block copolymers in a facile manner, as demonstrated here using binary blends, will likely be an important aspect of implementing directed assembly in nanomanufacturing.

Interestingly, the addition of only a small amount of the lower molecular-weight diblock copolymer in LB44 appears to reduce the overall range of substrate pattern periods over which defect-free lamellar domains form. Although nearperfect registration has been observed from $L_{S}=42.5 \mathrm{~nm}$ to $L_{S}=52.5 \mathrm{~nm}$ in the neat $L_{O 48}=48 \mathrm{~nm}$ block copolymer on patterned polystyrene brushes, ${ }^{19}$ the addition of $25 \%$ of the $L_{O 32}=32 \mathrm{~nm}$ block copolymer narrowed the range where perfect registration occurred to cover the range from $L_{S}$ $=42.5 \mathrm{~nm}$ to $L_{S}=47.5 \mathrm{~nm}$. This corresponds to a reduction in the commensurability range of substrate pattern periods that exhibit perfect pattern registration. In the LO48 block copolymer, perfect directed assembly has previously been shown to occur when $0.89<L_{S} / L_{O}<1.09$. For LB44 perfect directed assembly only occurred when $0.95<L_{S} / L_{O}<1.06$.

This reduction in the range of commensurability that directs the defect-free registration of block copolymer domains may be due to a higher-energy conformation of the binary
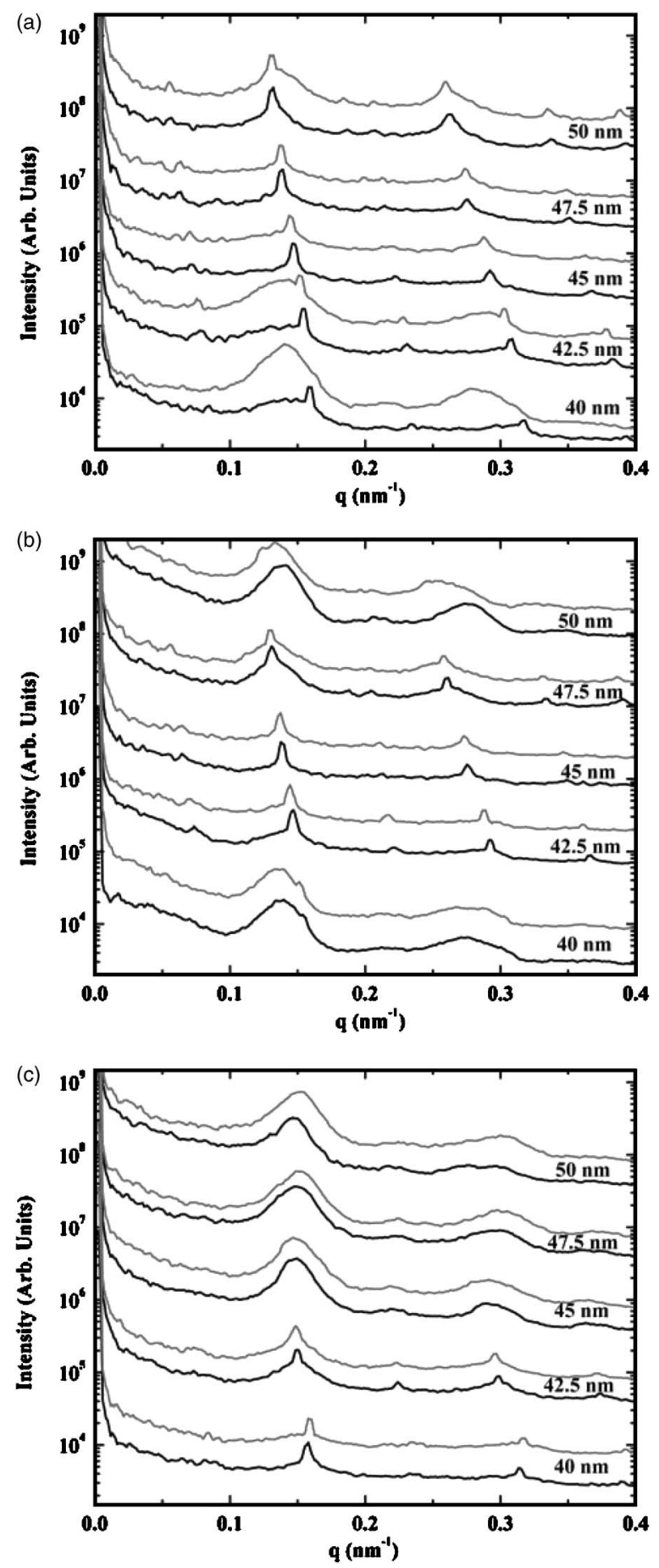

FIG. 3. Azimuthally averaged power spectra of 2D-FFTs of the scanning electron micrographs of block copolymer domains on chemically nanopatterned polystyrene and chemically nanopatterned neutral brushes. Black lines are for the block copolymer domain behavior on patterned polystyrene brushes; gray lines are for the block copolymer domain behavior on patterned neutral brushes. (a) LO48, (b) LB44, and (c) LB41. Substrate pattern periods are presented at the right side of the graphs. Spectra are offset for clarity.

blends. Others have previously studied the spatial distribution of the long and short chains in bulk binary blends of block copolymers and have found that the higher molecularweight block copolymers are stretched at the styrene-methyl 
methacrylate interface and are correspondingly compressed in the center of the domains to achieve a constant segmental density. ${ }^{22,27}$ We have previously shown, using phenomenological modeling, that the elastic free energy of stretching is a vital factor in achieving perfectly registered block copolymer domains on chemically nanopatterned substrates. ${ }^{19}$ Thus, the block copolymer blends, which may already be in a higher-energy stretched state, could potentially have much larger penalties for stretching their domains to a given distance, relative to the penalties required to stretch a neat copolymer with the same bulk lamellar period to that distance. This may explain the observed reduction in the improvement of the blend domain registration in response to increased interfacial energy contrast.

\section{CONCLUSIONS}

Binary blends of diblock copolymers serve as an effective means to achieve multiple length scales in the directed selfassembly of block copolymer thin films on chemically nanopatterned substrates. The substrate pattern period over which perfect domain registration occurs in the self-assembled binary blends of block copolymers was shifted between the bulk lamellar periods of two pure block copolymers. In all cases perfect directed assembly occurred on chemical surface patterns that had periods that were commensurate with the bulk lamellar period of the blend. These results relax the synthetic requirements of using directed assembly of block copolymer thin films for nanomanufacturing.

\section{ACKNOWLEDGMENTS}

Exposures were preformed on the $\mathrm{x}$-ray interference beamline at the Swiss Light Source, part of the Paul Scherrer Institut in Villigen, Switzerland. This research was supported by the Semiconductor Research Corporation (SRC) (2005OC-985), the National Science Foundation through the Nanoscale Science and Engineering Center (DMR-0425880), and the Camille Dreyfus Teacher-Scholar Award. This work is based in part upon research conducted at the Synchrotron Radiation Center, University of Wisconsin-Madison, which is supported by the NSF under Award No. DMR-0084402. One of the authors (M.P.S.) acknowledges a fellowship from the Semiconductor Research Corporation.

Presented at the 49th International Conference on Electron, Ion, and Photon Beam Technology and Nanofabrication, Orlando, Florida, 31 May-3 June 2005
${ }^{1}$ F. S. Bates and G. H. Fredrickson, Phys. Today 52, 32 (1999).

${ }^{2}$ M. Park, C. Harrison, P. M. Chaikin, R. A. Register, and D. H. Adamson, Science 276, 1401 (1997).

${ }^{3}$ R. R. Li, P. D. Dapkus, M. E. Thompson, W. G. Jeong, C. Harrison, P. M. Chaikin, R. A. Register, and D. H. Adamson, Appl. Phys. Lett. 76, 1689 (2000).

${ }^{4}$ C. T. Black, K. W. Guarini, K. R. Milkove, S. M. Baker, T. P. Russell, and M. T. Tuominen, Appl. Phys. Lett. 79, 409 (2001).

${ }^{5}$ C. T. Black, K. W. Guarini, Y. Zhang, H. J. Kim, J. Benedict, E. Sikorski, I. V. Babich, and K. R. Milkove, IEEE Electron Device Lett. 25, 622 (2004).

${ }^{6}$ T. Thurn-Albrecht, J. Schotter, C. A. Kastle, N. Emley, T. Shibauchi, L. Krusin-Elbaum, K. Guarini, C. T. Black, M. T. Tuominen, and T. P. Russell, Science 290, 2126 (2000).

${ }^{7}$ W. A. Lopes and H. M. Jaeger, Nature (London) 414, 735 (2001).

${ }^{8}$ K. Naito, H. Hieda, M. Sakurai, Y. Kamata, and K. Asakawa, IEEE Trans. Magn. 38, 1949 (2002).

${ }^{9}$ A. M. Urbas, M. Maldovan, P. DeRege, and E. L. Thomas, Adv. Mater. (Weinheim, Ger.) 14, 1850 (2002).

${ }^{10}$ D. E. Angelescu, J. H. Waller, D. H. Adamson, P. Deshpande, S. Y. Chou, R. A. Register, and P. M. Chaikin, Adv. Mater. (Weinheim, Ger.) 16, 1736 (2004).

${ }^{11}$ S. O. Kim, H. H. Solak, M. P. Stoykovich, N. J. Ferrier, J. J. de Pablo, and P. F. Nealey, Nature (London) 424, 411 (2003).

${ }^{12}$ S. H. Kim, M. J. Misner, T. Xu, M. Kimura, and T. P. Russell, Adv. Mater. (Weinheim, Ger.) 16, 226 (2004).

${ }^{13}$ H. W. Li and W. T. S. Huck, Nano Lett. 4, 1633 (2004).

${ }^{14}$ P. Mansky, J. DeRouchey, T. P. Russell, J. Mays, M. Pitsikalis, T. Morkved, and H. Jaeger, Macromolecules 31, 4399 (1998).

${ }^{15}$ T. L. Morkved, M. Lu, A. M. Urbas, E. E. Ehrichs, H. M. Jaeger, P. Mansky, and T. P. Russell, Science 273, 931 (1996).

${ }^{16}$ R. A. Segalman, H. Yokoyama, and E. J. Kramer, Adv. Mater. (Weinheim, Ger.) 13, 1152 (2001).

${ }^{17}$ D. Sundrani, S. B. Darling, and S. J. Sibener, Langmuir 20, 5091 (2004).

${ }^{18}$ D. Sundrani, S. B. Darling, and S. J. Sibener, Nano Lett. 4, 273 (2004).

${ }^{19}$ E. W. Edwards, M. F. Montague, H. H. Solak, C. J. Hawker, and P. F. Nealey, Adv. Mater. (Weinheim, Ger.) 16, 1315 (2004).

${ }^{20}$ M. P. Stoykovich, M. Müller, S. O. Kim, H. H. Solak, E. W. Edwards, J. J. de Pablo, and P. F. Nealey, Science 308, 1442 (2005).

${ }^{21}$ T. Hashimoto, Macromolecules 15, 1548 (1982).

${ }^{22}$ A. M. Mayes, T. P. Russell, V. R. Deline, S. K. Satija, and C. F. Majkrzak, Macromolecules 27, 7447 (1994).

${ }^{23}$ P. Mansky, Y. Liu, E. Huang, T. P. Russell, and C. Hawker, Science 275, 1458 (1997).

${ }^{24}$ H. H. Solak, C. David, J. Gobrecht, V. Golovkina, F. Cerrina, S. O. Kim, and P. F. Nealey, Microelectron. Eng. 67-68, 56 (2003).

${ }^{25}$ K. M. Pellerin, F. J. Himpsel, E. W. Edwards, and P. F. Nealey (unpublished).

${ }^{26}$ C. Harrison, P. M. Chaikin, D. A. Huse, R. A. Register, D. H. Adamson, A. Daniel, E. Huang, P. Mansky, T. P. Russell, C. J. Hawker, D. A. Egolf, I. V. Melnikov, and E. Bodenschatz, Macromolecules 33, 857 (2000).

${ }^{27}$ N. Koneripalli, R. Levicky, F. S. Bates, M. W. Matsen, S. K. Satija, J. Ankner, and H. Kaiser, Macromolecules 31, 3498 (1998). 